\title{
High rebleeding risk associated with choroidal collateral vessels in hemorrhagic moyamoya disease: analysis of a nonsurgical cohort in the Japan Adult Moyamoya Trial
}

\author{
Takeshi Funaki, MD, PhD, ${ }^{1}$ Jun C. Takahashi, MD, PhD, ${ }^{2}$ Kiyohiro Houkin, MD, PhD, ${ }^{3}$ \\ Satoshi Kuroda, MD, PhD, ${ }^{4}$ Shigekazu Takeuchi, MD, PhD, ${ }^{5}$ Miki Fujimura, MD, PhD, ${ }^{6}$ \\ Yasutake Tomata, $\mathrm{PhD},{ }^{7}$ and Susumu Miyamoto, MD, $\mathrm{PhD},{ }^{1}$ on behalf of the JAM Trial \\ Investigators
}

\begin{abstract}
${ }^{1}$ Department of Neurosurgery, Kyoto University Graduate School of Medicine, Kyoto; ${ }^{2}$ Department of Neurosurgery, National Cerebral and Cardiovascular Center, Suita; ${ }^{3}$ Department of Neurosurgery, Hokkaido University Graduate School of Medicine, Sapporo; " Department of Neurosurgery, Graduate School of Medicine and Pharmaceutical Sciences, University of Toyama; ${ }^{5}$ Department of Neurosurgery, Nagaoka Chuo General Hospital, Nagaoka; ${ }^{6}$ Department of Neurosurgery, Tohoku University Graduate School of Medicine, Sendai; and 'Division of Epidemiology, Department of Health Informatics and Public Health, Tohoku University School of Public Health, Graduate School of Medicine, Sendai, Japan
\end{abstract}

\begin{abstract}
OBJECTIVE Choroidal collateral vessels typical of moyamoya disease have received attention as a potential bleeding source. The authors' previous angiographic cross-sectional analysis suggested a possible association between choroidal collaterals and posterior hemorrhage, indicating a high risk for rebleeding. The present longitudinal analysis is intended to determine whether choroidal collaterals are a predictor of rebleeding in hemorrhagic moyamoya disease.

METHODS The Japan Adult Moyamoya Trial group designed an ancillary cohort study using 5-year follow-up data on 37 patients included in the nonsurgical arm of the original randomized controlled trial and compared the rebleeding rate of those with and those without choroidal collaterals, represented by the connection between the anterior or posterior choroidal arteries and the medullary arteries. An expert panel determined whether a choroidal collateral was present in each patient through the measurement of baseline angiography studies. The rebleeding rate comparison was adjusted for age, diagnosis of hypertension, and involvement of the posterior cerebral artery.
\end{abstract}

RESULTS Choroidal collaterals were present in 21 patients (56.8\%). The rebleeding rate was $13.1 \%$ per year in the collateral-positive group as compared with $1.3 \%$ in the negative group ( $p=0.008$, log-rank test). The adjusted hazard ratio for rebleeding in the collateral-positive group relative to the negative group remained statistically significant (HR 11.10, $95 \% \mathrm{Cl} 1.37-89.91$ ). Radiographic assessment of the collateral-positive group revealed good correspondence between the distribution of collaterals and rebleeding sites.

CONCLUSIONS Results of this study suggest that choroidal collaterals are a bleeding source with a high risk for hemorrhagic recurrence and a predictor of rebleeding in hemorrhagic moyamoya disease.

https://thejns.org/doi/abs/10.3171/2017.9.JNS17576

KEY WORDS moyamoya disease; intracerebral hemorrhage; choroidal artery; cohort studies; natural history; vascular disorders

I NTRACRANIAL hemorrhage is a common symptom of adult moyamoya disease. The high incidence of rebleeding after initial hemorrhage, approximately $7 \%$ per year, ${ }^{9,18}$ is a major concern with this disease. Researchers have identified only a few risk factors for such rebleeding. ${ }^{18}$ In an earlier study, we demonstrated that posterior hemorrhage-defined as an initial hemorrhage occurring in the posterior half of the brain such as the thalamus or atrium-is a strong predictor of rebleeding; ${ }^{23}$ however, the pathological mechanism of the high rebleeding risk related to posterior hemorrhage remains unknown.

The various abnormal, fragile collaterals typical of moyamoya disease are candidates for the underlying cause of bleeding, and those derived from the choroidal arter-

ABBREVIATIONS HR = hazard ratio; JAM = Japan Adult Moyamoya; $m R S=$ modified Rankin Scale; PCA = posterior cerebral artery.

SUBMITTED March 6, 2017. ACCEPTED September 1, 2017.

INCLUDE WHEN CITING Published online March 2, 2018; DOI: 10.3171/2017.9.JNS17576. 

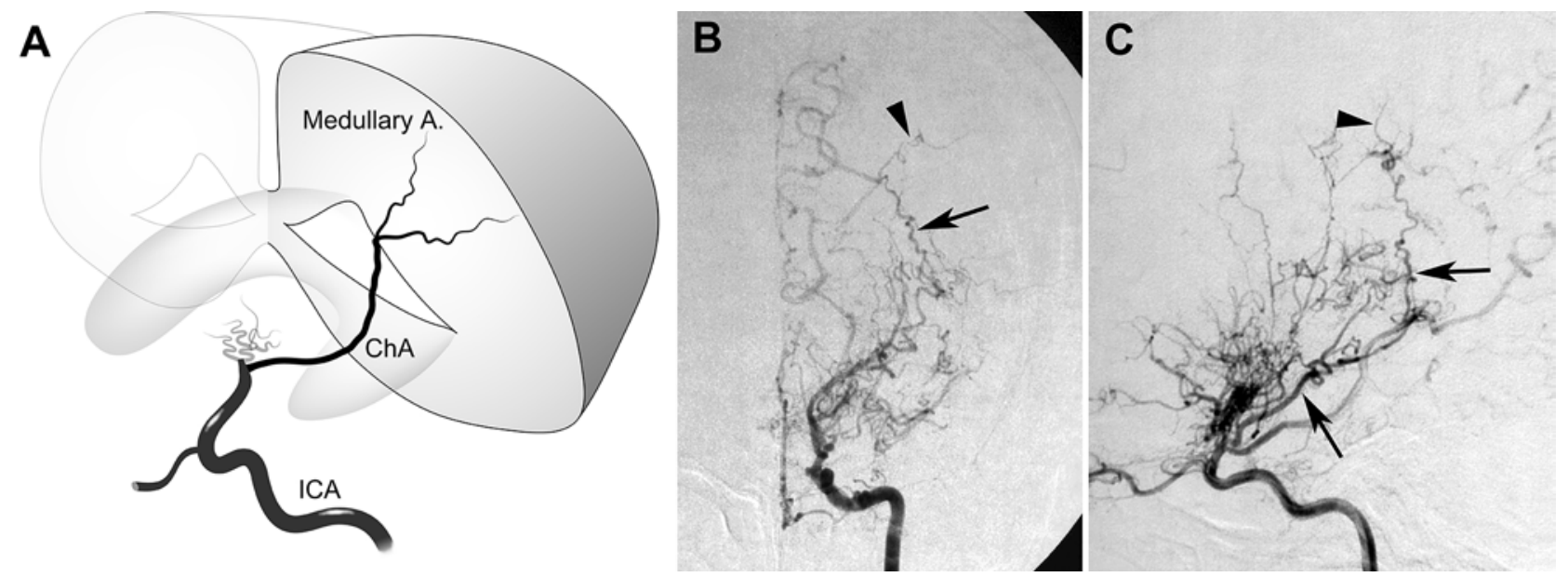

FIG. 1. Illustration (A) and representative angiograms, anteroposterior (B) and lateral (C) views, showing choroidal anastomosis. Dilated and extended anterior or posterior choroidal artery (arrows) connects to the medullary artery (arrowheads). Although the illustration represents choroidal anastomosis from the anterior choroidal artery, together the anterior choroidal artery from the internal carotid artery and the lateral posterior choroidal arteries from the PCA can serve as the origin of such an anastomosis. $\mathrm{A}$. = artery; $\mathrm{ChA}=$ choroidal artery; ICA = internal carotid artery. Illustration copyright Takeshi Funaki. Published with permission.

ies have received special attention. Several case series and cross-sectional studies have suggested an association between the presence of choroidal collaterals and hemorrhagic presentation. ${ }^{6,7,15,17,19}$ According to our angiographic cross-sectional analyses, choroidal collaterals are most likely responsible for posterior hemorrhage. ${ }^{3}$ In longitudinal studies, however, the association between choroidal collaterals and rebleeding has not been sufficiently documented. The aim of the present study is to determine, from longitudinal data on the nonsurgical cohort of the Japan Adult Moyamoya (JAM) Trial, whether choroidal collateral vessels observed on baseline angiography are predictors of rebleeding in hemorrhagic moyamoya disease.

\section{Methods}

\section{Participants and Setting}

The JAM Trial was a multicenter randomized controlled trial undertaken to test the hypothesis that direct bypass surgery reduces the incidence of rebleeding in hemorrhagic moyamoya disease. The study was registered with the UMIN Clinical Trials Registry (www.umin.ac.jp/ ctr/index.htm), and its registration no. is C000000166. The primary report on the trial showed a significant difference between the surgical and nonsurgical groups in a Kaplan-Meier analysis, suggesting that direct bypass has a preventive effect on rebleeding..$^{6}$ We designed an ancillary cohort study using the longitudinal data on patients allocated to the nonsurgical arm. As the research question behind the present analysis was raised during our previous analyses,, 323 an expert panel developed the current study design after the completion of patient follow-ups. The JAM Trial recruited Japanese patients from 22 centers who had been diagnosed with moyamoya disease according to the guidelines proposed by the Ministry of Health and Welfare of Japan, ${ }^{1}$ had experienced intracranial hemorrhage within the 12 months before randomization, were ages 16-65 years, were independent in daily life (modi- fied Rankin Scale [mRS] score 0-2), had completed acute phase treatment at least 1 month before randomization, and had been free from ischemic or hemorrhagic attack for at least 1 month. ${ }^{16}$ Among the 213 patients assessed for eligibility, $80(37.6 \%)$ were enrolled in the trial. Thirtyeight patients were assigned to the nonsurgical group and were prescribed blood pressure medication if required. The administration of anticoagulants or antiplatelet drugs was prohibited unless the patient had been experiencing significant cerebral ischemic attacks. One neurologist and one neurosurgeon at each institute followed each patient. The study protocol required that each patient undergo a 5-year scheduled follow-up.

\section{Variables of Interest}

The choroidal collateral vessel, the primary variable of interest, was anatomically defined as anastomosis between the choroidal artery and the medial end of the medullary artery (choroidal anastomosis; Fig. 1A), a phenomenon typical of the disease and serving as a supply to the cortex..$^{2,5}$ Together the anterior choroidal artery from the internal carotid artery and the lateral posterior choroidal arteries from the posterior cerebral artery (PCA) can serve as the origin of such an anastomosis. The presence or absence of this collateral was assessed with baseline internal carotid artery and vertebral artery conventional digital subtraction angiography performed during the 120 days before study enrollment in all cases. A positive indicator of choroidal anastomosis in the lateral angiographic view is extreme dilation and extension of the anterior or lateral posterior choroidal artery with sudden deviation from the shape of the lateral ventricle at its peripheral portion to connect to the medullary artery; in the anteroposterior view, this collateral has a typical sharp inflection to the lateral (Fig. 1B and C). Extreme extension of the anterior choroidal or lateral posterior choroidal artery toward the body of the ventricle and the connection between the medial posterior choroidal artery and the pericallosal ar- 
tery were also considered indicators. These angiographic definitions had been developed by the trial group in previous angiographic cross-sectional analyses. ${ }^{3}$

An expert panel assembled for the present project identified the presence of anastomosis in each hemisphere by using baseline angiography, and this identification was performed after completion of the patient follow-ups. The variable of choroidal anastomosis was rated as "positive" when present in at least one hemisphere or "negative" when absent in both hemispheres. To minimize information bias in the assessment of angiographic variables, all members of the panel were blinded to whether rebleeding had occurred.

In light of the study hypothesis, patient age and diagnosis of hypertension were considered potential confounders. The location of initial bleeding, defined as either anterior or posterior, was considered an intervening variable on the causal pathway between choroidal collaterals and rebleeding because the location of bleeding was likely to be highly dependent on the type of collateral; therefore, this variable was excluded from statistical models. Instead, we included involvement of the PCA as a potential confounder. In the present analysis, PCA involvement was defined as stenosis greater than $50 \%$ in the $\mathrm{P}_{1}$ to $\mathrm{P}_{3}$ segment of either PCA with decreased delineation of the cortical arteries in either hemisphere measured with angiography at onset. ${ }^{4}$

All variables except for angiographic variables were recorded at patient enrollment, including diagnosis of hypertension, diabetes mellitus, and hyperlipidemia and history of ischemic and hemorrhagic events.

The outcome variable of interest was a rebleeding attack occurring more than 3 months after enrollment and related to death or significant morbidity defined as an mRS score exceeding 2 . This variable was a secondary end point in the original randomized controlled trial. ${ }^{16}$

\section{Statistical Analysis}

The t-test, chi-square test, or Fisher's exact test was used for comparison of the baseline characteristics. The log-rank test was used to compare the time to an event between groups. The effect of choroidal anastomosis on rebleeding was estimated as a hazard ratio (HR) calculated with Cox proportional hazards regression and adjusted for 3 potential confounders: patient age, diagnosis of hypertension, and PCA involvement.

Of the 38 nonsurgical cases, 1 patient could not have baseline angiography assessed because the hospital performing the angiography had lost the original films and was thus excluded from the primary analysis. The patient in this case suffered rebleeding 59 months after enrollment. In addition to the primary analysis that excluded this case, we conducted a sensitivity analysis that included this case by complementing the missing data. As the data on choroidal anastomosis and PCA involvement were missing in this case, the multiple imputation method was adopted for these 2 variables for the sensitivity Cox regression analysis. With this method, 5 imputed data sets were generated with the SPSS command "MULTIPLE IMPUTATION" using the "Automatic" option as the imputation method. These 5 imputed data sets were used for the pooled Cox regression. Two-sided values of $\mathrm{p}<0.05$
TABLE 1. Baseline variables in 37 moyamoya patients in the nonsurgical group

\begin{tabular}{lccr}
\hline \multirow{2}{*}{ Variable } & \multicolumn{2}{c}{ Choroidal Anastomosis } & \multirow{2}{p}{} \\
\cline { 2 - 3 } & Positive & Negative & Value \\
\hline No. of patients & 21 & 16 & \\
\hline Mean age in yrs \pm SD & $39.9 \pm 13.4$ & $43.4 \pm 11.1$ & 0.405 \\
\hline Female & $17(81.0)$ & $10(62.5)$ & 0.274 \\
\hline Hypertension & $4(19.0)$ & $3(18.8)$ & 1.000 \\
\hline Diabetes mellitus & $1(4.8)$ & $1(6.3)$ & 1.000 \\
\hline Hyperlipidemia & $1(4.8)$ & $1(6.3)$ & 1.000 \\
\hline Hx of ischemic event & $7(33.3)$ & $3(18.8)$ & 0.461 \\
\hline Hx of hemorrhagic stroke & $2(9.5)$ & $2(12.5)$ & 1.000 \\
\hline PCA involvement & $9(42.9)$ & $3(18.8)$ & 0.121 \\
\hline
\end{tabular}

$\mathrm{Hx}=$ history.

Values expressed as number (\%) of patients, unless otherwise indicated.

and 95\% CIs not including 1 were considered significant. All analyses were performed with IBM SPSS Statistics Desktop, version 22 (IBM Corp.).

As a supplementary analysis, the incidence of rebleeding was compared between the nonsurgical and surgical groups within the choroidal collateral-positive stratum. This analysis was performed in response to peer reviews. In the original randomized controlled trial, the participants assigned to the surgical group underwent extracranial-intracranial bypass, a direct anastomotic procedure such as superficial temporal artery-middle cerebral artery anastomosis. They had been followed for 5 years in the same fashion as those assigned to the nonsurgical group.

\section{Results \\ Analyses of Nonsurgical Cohort}

Of the 37 patients analyzed, $21(56.8 \%)$ were identified as positive for choroidal anastomosis, whereas $16(43.2 \%)$ were identified as negative. Involvement of the PCA was detected in 12 patients (32.4\%). Table 1 summarizes the baseline characteristics of patients with and without choroidal anastomosis. Although the relatively small study population might weaken statistical significance, no significant differences in age; sex; diagnosis of hypertension, diabetes mellitus, or hyperlipidemia; history of ischemic or hemorrhagic event; or PCA involvement were observed between the 2 groups.

All patients completed the scheduled follow-up, and no loss to follow-up occurred. Rebleeding occurred in 11 (29.7\%) of the 37 cases. The overall incidence of rebleeding calculated with the person-year method was 11/153.27 or $7.2 \%$ per year.

Kaplan-Meier analysis revealed that the incidence of rebleeding was significantly higher in the choroidal anastomosis-positive group than in the negative group ( $\mathrm{p}=$ 0.008 ; Fig. 2). The incidence of rebleeding in those with and those without choroidal anastomosis was $13.1 \%$ and $1.3 \%$ per year, respectively. The 5-year risk of rebleeding reached $47.6 \%$ for those with choroidal anastomosis and $6.3 \%$ for those without. The HR for rebleeding in the choroidal anastomosis-positive group relative to the negative 


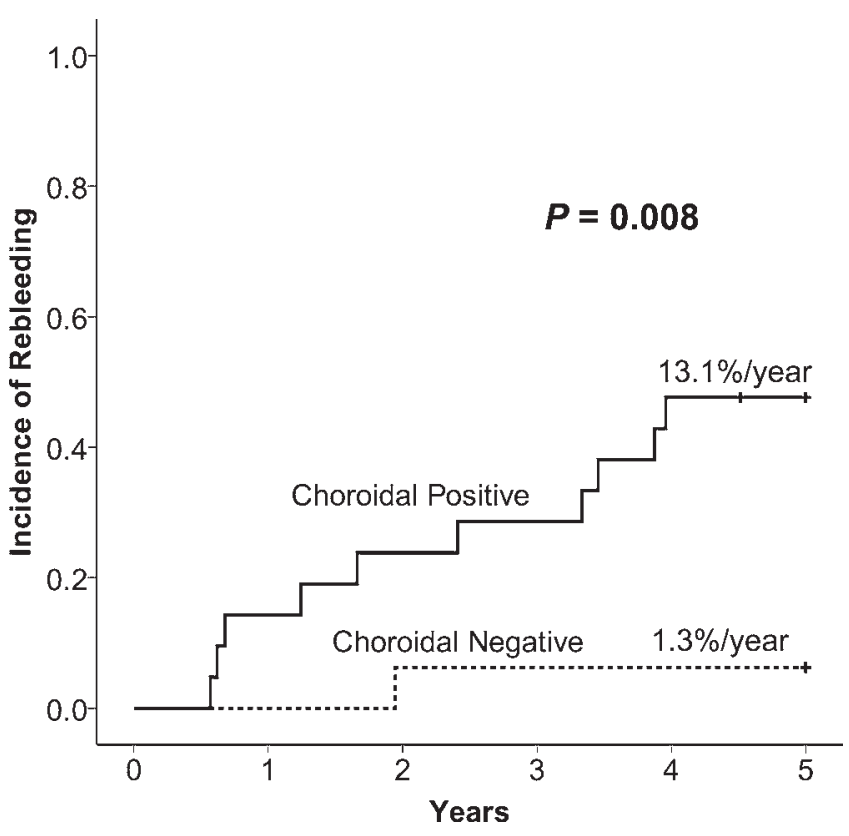

FIG. 2. Kaplan-Meier curve for rebleeding (37 patients).

group remained statistically significant after adjustments for age, diagnosis of hypertension, and PCA involvement (HR 11.10, 95\% CI 1.37-89.91; Table 2). Sensitivity analysis, which included the 1 case with missing angiography data, also revealed statistical significance in the Cox proportional hazards regression.

Table 3 summarizes radiographic characteristics of 10 patients positive for choroidal anastomosis and rebleeding attack. In 7 patients, choroidal anastomosis was reasonably considered to be responsible for the rebleeding because the rebleeding occurred in the hemisphere in which choroidal anastomosis was present and corresponded to the distribution of choroidal arteries, including the periventricular area around the atrium or the posterior part of the body of the lateral ventricle.

\section{Supplementary Analysis}

Of the 80 participants included in the original randomized controlled trial, 45 were identified as positive for choroidal anastomosis; this population included 24 surgical and 21 nonsurgical cases. The analysis for this subgroup revealed a significantly lower rebleeding rate in the surgical group than in the nonsurgical group on multivariate analysis (HR 0.33, 95\% CI 0.11-0.99; Table 4).

\section{Discussion}

Results of the present cohort study suggest that choroidal anastomosis is an independent predictor of rebleeding in hemorrhagic moyamoya disease. The good correspondence between the distribution of collateral vessels and rebleeding sites suggests that choroidal anastomoses are responsible for most rebleeding events.

The overall incidence of rebleeding in the present study cohort (7.2\% per year) is similar to findings in other reports; this concordance may represent minimal contami-
TABLE 2. Multiple-adjusted hazard ratios for rebleeding in primary and sensitivity analyses

\begin{tabular}{|c|c|c|c|c|}
\hline \multirow[b]{2}{*}{ Analysis } & \multicolumn{2}{|r|}{ Crude } & \multicolumn{2}{|c|}{ Multivariate Adjustment* } \\
\hline & $\mathrm{HR}$ & $95 \% \mathrm{Cl}$ & HR & $95 \% \mathrm{Cl}$ \\
\hline \multicolumn{5}{|c|}{$\begin{array}{l}\text { Choroidal anastomosis } \\
\text { (primary analysis) } \dagger\end{array}$} \\
\hline Negative & 1.00 & Reference & 1.00 & Reference \\
\hline Positive & 9.66 & $1.23-75.60$ & 11.10 & $1.37-89.91$ \\
\hline \multicolumn{5}{|c|}{$\begin{array}{l}\text { Choroidal anastomosis } \\
\text { (sensitivity analysis) } \ddagger\end{array}$} \\
\hline Negative & 1.00 & Reference & 1.00 & Reference \\
\hline Positive & 9.10 & $1.16-71.59$ & 10.82 & $1.33-88.35$ \\
\hline
\end{tabular}

nation with selection bias in the present study. Kobayashi et al. reported that the rebleeding incidence reached 7.09\%/ person/year in hemorrhagic moyamoya disease. ${ }^{9}$ Although Morioka et al. did not calculate rebleeding incidence in their cohort study, the incidence could reach 6.5\%/person/ year according to our calculation using their figures. ${ }^{18}$

The present cohort study confirms the results of pioneering cross-sectional studies suggesting the significance of choroidal collateral vessels as risk factors for rebleeding. Irikura et al. reported that the prevalence of enlarged choroidal arteries and medullary arteries was higher in hemorrhagic moyamoya disease than in ischemic disease. ${ }^{6}$ Morioka et al. also found, through angiographic crosssectional analyses of 107 patients with moyamoya disease, that dilation and branch extension of the anterior choroidal artery were seen more frequently in hemorrhagic patients than in ischemic or asymptomatic patients. ${ }^{17} \mathrm{~A}$ more recent pediatric series related to hemorrhagic moyamoya disease revealed a similar result with choroidal collaterals. ${ }^{12}$

Despite these similarities, cohort studies have rarely focused on the impact of the choroidal collateral vessel..$^{9,18}$ We found only 2 cohort studies assessing the vessel's impact on rebleeding. In their 2-year exploratory cohort study, Sun et al. found that the dilation and extension of anterior choroidal and posterior communicating arteries were associated with subsequent intraventricular hemorrhage, although the association was not statistically significant after adjustment. ${ }^{21}$ Their study differed from ours in several areas. First, they included a substantial number of patients with ischemic moyamoya disease, which presents a much lower risk of hemorrhage. ${ }^{24}$ Second, the follow-up period in their study was shorter than that in ours. Third, they considered collaterals from the anterior choroidal arteries and those from the posterior communicating artery as a single variable. Liu et al. analyzed follow-up data on 145 adults (290 hemispheres) with hemorrhagic moyamoya disease and concluded that the dilation was associated with initial hemorrhage but not with recurrence. ${ }^{13}$ The disagreement between their results and ours is attributable to the critical difference in study design. In the study by Liu et al., most of the patients (271/290 hemispheres) underwent indirect 
TABLE 3. Radiographic data on patients positive for choroidal anastomosis and rebleeding attack

\begin{tabular}{|c|c|c|c|c|c|}
\hline Case No. & $\begin{array}{l}\text { Age } \\
\text { (yrs) }\end{array}$ & Sex & $\begin{array}{l}\text { Choroidal } \\
\text { Anastomosis }\end{array}$ & $\begin{array}{c}\text { Time to } \\
\text { Rebleeding (yrs) }\end{array}$ & Rebleeding Site \\
\hline \multicolumn{6}{|c|}{$\begin{array}{l}\text { Rebleeding attributable to choroidal } \\
\text { anastomosis }\end{array}$} \\
\hline 1 & 37 & $\mathrm{~F}$ & Rt & 3.5 & Rt periventricular area of pst part of body of lat ventricle \\
\hline 2 & 31 & $\mathrm{~F}$ & Bilat & 2.4 & Lt periventricular area of atrium \\
\hline 3 & 54 & $\mathrm{~F}$ & Bilat & 3.3 & Lt periventricular area of atrium \\
\hline 4 & 32 & $\mathrm{~F}$ & Bilat & 0.6 & Rt periventricular area of pst part of body of lat ventricle \\
\hline 5 & 43 & $\mathrm{~F}$ & Bilat & 1.7 & Rt periventricular area of atrium \\
\hline 6 & 41 & $\mathrm{~F}$ & Rt & 4.0 & Rt periventricular area of atrium \\
\hline 7 & 37 & $\mathrm{~F}$ & Bilat & 3.9 & Rt periventricular area of atrium \\
\hline \multicolumn{6}{|c|}{$\begin{array}{l}\text { Rebleeding not attributable to choroidal } \\
\text { anastomosis }\end{array}$} \\
\hline 8 & 53 & $\mathrm{~F}$ & Rt & 1.2 & Lt thalamus \\
\hline 9 & 51 & $\mathrm{~F}$ & Rt & 0.6 & Rt anterior thalamus \\
\hline 10 & 62 & M & Rt & 0.7 & Lt head of caudate \\
\hline
\end{tabular}

pst $=$ posterior

bypass after baseline angiography. ${ }^{13}$ Bypass surgery itself could diminish the collateral from choroidal arteries; this possible phenomenon could result in an underestimation of the effect of choroidal collaterals. ${ }^{7}$ Another difference between the studies is the definition of "collateral"; our definition of choroidal anastomosis included assessment of the posterior choroidal artery while theirs did not.

A high rebleeding risk related to choroidal anastomosis could be attributable to fragile pathology in and an increased hemodynamic burden on the collateral. Recent studies employing high-resolution MR angiography have revealed that the collateral represents a connection between the choroidal and medullary arteries in the periventricular area. ${ }^{2,5}$ Radiographic evidence of microbleeds in the periventricular area in moyamoya disease could support the hypothesis of fragility at the connection site. ${ }^{2,8,20}$ These fragile connections could cause rupture from long exposure to the hemodynamic burden. The burden on the choroidal anastomosis could increase as the disease progresses. According to a stage classification representing the disease progression, ${ }^{22}$ basal collateral vessels formed by the perforating arteries from the anterior and middle cerebral arteries shrink as the terminal portion of the internal carotid artery becomes narrower. Thus, disease progression may shift the hemodynamic burden onto the choroidal anastomosis, resulting in rupture. The hemodynamic burden could also promote the formation of microaneurysms at the connection sites of the collaterals, which could represent another mechanism of bleeding. . $^{1011,14}$

Choroidal anastomosis explains well the mechanism of posterior hemorrhage at high risk for rebleeding. The anastomosis is distributed mainly in the periventricular area around the atrium or the posterior part of the body of the lateral ventricle. ${ }^{2}$ Rupture of a choroidal anastomosis could contribute to posteriorly distributed intraventricular hemorrhage, posterior thalamic hemorrhage, or posterior temporal lobe hemorrhage, all of which are defined as posterior hemorrhage. ${ }^{23} \mathrm{~A}$ high rebleeding risk related to posterior hemorrhage may be attributable to the extreme fragility of choroidal anastomosis distributed posteriorly.

The importance of choroidal anastomosis as a risk factor for rebleeding may be enhanced by the knowledge that the risk factor is treatable with bypass surgery? Its importance may also be enhanced by the results of our supplementary analysis, suggesting the benefit of bypass surgery in reducing the rebleeding rate in the choroidal anastomosis-positive subgroup. Further studies focused on collaterals may improve the effectiveness of bypass surgery in preventing rebleeding.

The present study has several limitations. First, the number of participants is relatively small. As hemorrhagic moyamoya disease is a rare condition, recruitment of patients is very difficult. Our study sample size was almost equal to those in previous cohort studies. ${ }^{9,18}$ Other larger follow-up studies have targeted a population different from ours: medically and surgically treated patients. ${ }^{13,21}$ Second, the reliability of the angiographic diagnosis of choroidal anastomosis remains an issue to be assessed. Third, as the present study addresses ancillary analyses of an original randomized controlled trial, the hypothesis of the present study differs from that originally specified in the trial. Newly designed cohort studies are required to confirm whether the present findings can be repeated.

TABLE 4. Supplementary analysis: multiple-adjusted hazard ratios for rebleeding in the choroidal anastomosis-positive subgroup

\begin{tabular}{lcccccc}
\hline \multirow{2}{*}{ Group } & No. of & & \multicolumn{2}{c}{ Crude } & & \multicolumn{2}{c}{ Multivariate Adjustment* } \\
\cline { 3 - 4 } \cline { 6 - 7 } & Patients & HR & $95 \% \mathrm{Cl}$ & & HR & $95 \% \mathrm{Cl}$ \\
\hline Nonsurgical & 21 & 1.00 & Reference & & 1.00 & Reference \\
\hline Surgical & 24 & 0.39 & $0.13-1.14$ & & 0.33 & $0.11-0.99$ \\
\hline
\end{tabular}

* Adjusted for age (continuous variable), hypertension (yes/no), and PCA involvement (yes/no). 


\section{Conclusions}

Results of the present cohort study support our hypothesis that choroidal collateral vessels are a predictor of rebleeding in hemorrhagic moyamoya disease.

\section{Acknowledgments}

The JAM Trial has been funded since 1999 by a grant from the Japanese Ministry of Health, Labour and Welfare as a major project of the Research Committee on Spontaneous Occlusion of the Circle of Willis (moyamoya disease).

\section{References}

1. Fukui M: Guidelines for the diagnosis and treatment of spontaneous occlusion of the circle of Willis ('moyamoya' disease). Clin Neurol Neurosurg 99 (Suppl 2):S238-S240, 1997

2. Funaki T, Fushimi Y, Takahashi JC, Takagi Y, Araki Y, Yoshida $\mathrm{K}$, et al: Visualization of periventricular collaterals in moyamoya disease with flow-sensitive black-blood magnetic resonance angiography: preliminary experience. Neurol Med Chir (Tokyo) 55:204-209, 2015

3. Funaki T, Takahashi JC, Houkin K, Kuroda S, Takeuchi S, Fujimura M, et al: Angiographic features of hemorrhagic moyamoya disease with high recurrence risk: a supplementary analysis of the Japan Adult Moyamoya Trial. J Neurosurg [epub ahead of print April 14, 2017. DOI: 10.3171/2016.11. JNS161650]

4. Funaki T, Takahashi JC, Takagi Y, Yoshida K, Araki Y, Kikuchi T, et al: Impact of posterior cerebral artery involvement on long-term clinical and social outcome of pediatric moyamoya disease. J Neurosurg Pediatr 12:626-632, 2013

5. Funaki T, Takahashi JC, Yoshida K, Takagi Y, Fushimi Y, Kikuchi T, et al: Periventricular anastomosis in moyamoya disease: detecting fragile collateral vessels with MR angiography. J Neurosurg 124:1766-1772, 2016

6. Irikura K, Miyasaka Y, Kurata A, Tanaka R, Fujii K, Yada K, et al: A source of haemorrhage in adult patients with moyamoya disease: the significance of tributaries from the choroidal artery. Acta Neurochir (Wien) 138:1282-1286, 1996

7. Jiang H, Ni W, Xu B, Lei Y, Tian Y, Xu F, et al: Outcome in adult patients with hemorrhagic moyamoya disease after combined extracranial-intracranial bypass. J Neurosurg 121:1048-1055, 2014

8. Kazumata K, Shinbo D, Ito M, Shichinohe H, Kuroda S, Nakayama N, et al: Spatial relationship between cerebral microbleeds, moyamoya vessels, and hematoma in moyamoya disease. J Stroke Cerebrovasc Dis 23:1421-1428, 2014

9. Kobayashi E, Saeki N, Oishi H, Hirai S, Yamaura A: Longterm natural history of hemorrhagic moyamoya disease in 42 patients. J Neurosurg 93:976-980, 2000

10. Kuroda S, Houkin K, Kamiyama H, Abe H: Effects of surgical revascularization on peripheral artery aneurysms in moyamoya disease: report of three cases. Neurosurgery 49:463-468, 2001

11. Lee JK, Lee JH, Kim SH, Lee MC: Distal anterior choroidal artery aneurysm in a patient with moyamoya disease: case report. Neurosurgery 48:222-225, 2001

12. Liu P, Han C, Li DS, Lv XL, Li YX, Duan L: Hemorrhagic moyamoya disease in children: clinical, angiographic features, and long-term surgical outcome. Stroke 47:240-243, 2016

13. Liu P, Liu AH, Han C, Chen C, Lv XL, Li DS, et al: Difference in angiographic characteristics between hemorrhagic and nonhemorrhagic hemispheres associated with hemorrhage risk of moyamoya disease in adults: a self-controlled study. World Neurosurg 95:348-356, 2016
14. Liu P, Lv XL, Liu AH, Chen C, Ge HJ, Jin HW, et al: Intracranial aneurysms associated with moyamoya disease in children: clinical features and long-term surgical outcome. World Neurosurg 94:513-520, 2016

15. Liu W, Zhu S, Wang X, Yue X, Zhou Z, Wang H, et al: Evaluation of angiographic changes of the anterior choroidal and posterior communicating arteries for predicting cerebrovascular lesions in adult moyamoya disease. J Clin Neurosci 18:374-378, 2011

16. Miyamoto S, Yoshimoto T, Hashimoto N, Okada Y, Tsuji I, Tominaga T, et al: Effects of extracranial-intracranial bypass for patients with hemorrhagic moyamoya disease: results of the Japan Adult Moyamoya Trial. Stroke 45:1415-1421, 2014

17. Morioka M, Hamada J, Kawano T, Todaka T, Yano S, Kai Y, et al: Angiographic dilatation and branch extension of the anterior choroidal and posterior communicating arteries are predictors of hemorrhage in adult moyamoya patients. Stroke 34:90-95, 2003

18. Morioka M, Hamada J, Todaka T, Yano S, Kai Y, Ushio Y: High-risk age for rebleeding in patients with hemorrhagic moyamoya disease: long-term follow-up study. Neurosurgery 52:1049-1055, 2003

19. Nah HW, Kwon SU, Kang DW, Ahn JS, Kwun BD, Kim JS: Moyamoya disease-related versus primary intracerebral hemorrhage: location and outcomes are different. Stroke 43:1947-1950, 2012 (Erratum in Stroke 44:e119, 2013)

20. Qin Y, Ogawa T, Fujii S, Shinohara Y, Kitao S, Miyoshi F, et al: High incidence of asymptomatic cerebral microbleeds in patients with hemorrhagic onset-type moyamoya disease: a phase-sensitive MRI study and meta-analysis. Acta Radiol 56:329-338, 2015

21. Sun W, Yuan C, Liu W, Li Y, Huang Z, Zhu W, et al: Asymptomatic cerebral microbleeds in adult patients with moyamoya disease: a prospective cohort study with 2 years of follow-up. Cerebrovasc Dis 35:469-475, 2013

22. Suzuki J, Kodama N: Moyamoya disease-a review. Stroke 14:104-109, 1983

23. Takahashi JC, Funaki T, Houkin K, Inoue T, Ogasawara K, Nakagawara J, et al: Significance of the hemorrhagic site for recurrent bleeding: prespecified analysis in the Japan Adult Moyamoya Trial. Stroke 47:37-43, 2016

24. Yamada S, Oki K, Itoh Y, Kuroda S, Houkin K, Tominaga T, et al: Effects of surgery and antiplatelet therapy in ten-year follow-up from the Registry Study of Research Committee on Moyamoya Disease in Japan. J Stroke Cerebrovasc Dis 25:340-349, 2016

\section{Disclosures}

The authors report no conflict of interest concerning the materials or methods used in this study or the findings specified in this paper.

\section{Author Contributions}

Conception and design: Miyamoto, Funaki. Acquisition of data: Miyamoto, Funaki, Takahashi, Kuroda, Takeuchi, Fujimura. Analysis and interpretation of data: all authors. Drafting the article: Funaki. Critically revising the article: all authors. Reviewed submitted version of manuscript: all authors. Approved the final version of the manuscript on behalf of all authors: Miyamoto. Statistical analysis: Tomata.

\section{Correspondence}

Susumu Miyamoto: Kyoto University Graduate School of Medicine, Sakyo-ku, Kyoto, Japan. miy@kuhp.kyoto-u.ac.jp. 